\title{
The effects of interactivity and product information on consumers' emotional responses to an online retail setting
}

\author{
Department of Management \\ University of Canterbury \\ Private Bag 4800, Christchurch, New Zealand \\ Fax: +64 33642020 \\ E-mail: paul.ballantine@canterbury.ac.nz \\ E-mail: david.fortin@canterbury.ac.nz \\ *Corresponding author
}

Paul W. Ballantine* and David R. Fortin

\begin{abstract}
This study examined how two aspects of an online shopping environment can influence the emotional states of consumers, and how these emotions subsequently affect their intentions to purchase products online. A web-based experiment (using a $3 \times 3$ between-subjects factorial design) was conducted, where respondents were exposed to a simulated online retail store. A total of 360 responses were collected from web users. The two independent variables examined were level of interactivity and amount of information. Emotion was measured using the two emotional states of pleasure and arousal. Results suggest that a linear relationship exists between the level of interactivity provided by an online store and pleasure. Moreover, pleasure (and in some cases arousal) was found to be a significant predictor of the likelihood that a consumer would purchase products online.
\end{abstract}

Keywords: online shopping; interface design; consumer behaviour; emotion.

Reference to this paper should be made as follows: Ballantine, P.W. and Fortin, D.R. ( $\mathrm{xxxx)}$ 'The effects of interactivity and product information on consumers' emotional responses to an online retail setting', Int. J. Internet Marketing and Advertising, Vol. X, No. Y, pp.000-000.

Biographical notes: Paul W. Ballantine is a Senior Lecturer in Marketing at the University of Canterbury in Christchurch, New Zealand. $\mathrm{He}$ was formerly based at the University of Auckland, before returning to his home city of Christchurch. He has published in outlets including Advances in Consumer Research and the International Journal of Retail and Distribution Management, and has presented at numerous international conferences. His research interests include retailing, social and ethical issues in marketing, research methodologies and statistics, consumer behaviour and digital commerce.

David R. Fortin is an Associate Professor in Marketing and part of the Department of Management at the University of Canterbury in New Zealand. He has a PhD in Marketing from the University of Rhode Island, USA and his research has been published in outlets such as the Journal of Advertising Research, International Journal of Mobile Marketing, Journal of Business Research, Psychology \& Marketing, International Journal of Entrepreneurship 
and Innovation Management, British Food Journal and Telematics \& Informatics. He is the Director of the online experimental consumer research project 'Web-Lab' at the University of Canterbury. His research focus is in the area of interactive marketing and e-commerce, consumer research on the web, advertising effectiveness, research methodology, genetically modified foods, and attitude change and formation.

\section{Introduction}

With increasing awareness that the configuration of a web-based shopping interface may provide a source of competitive differentiation, it is important to consider how the various elements of an online environment can affect the purchasing behaviour of consumers. In traditional bricks-and-mortar settings, research concerning the effects of retail environments on consumer behaviour owe much to the work of Kotler (1974). Coining the term "atmospherics", Kotler (1974) argued that buying environments can be purposefully designed to produce specific emotional effects in shoppers, thereby enhancing their purchase probability. Although research about the effects of emotion in traditional retail environments is indebted to the work of Kotler, it was not until Donovan and Rossiter (1982) that research in this area was popularised. In their paper, the authors introduced the Mehrabian-Russell framework (Mehrabian and Russell, 1974), suggesting that it provides a parsimonious description of environments, intervening variables and behaviours that are relevant to retail settings. Using this framework, several authors (e.g., Baker et al., 1992; Donovan et al., 1994; Sherman et al., 1997) have investigated how retail environments can affect the emotional states and subsequent purchasing behaviour of consumers.

Found to be an important factor in bricks-and-mortar stores, emotion may also play an integral role in helping understand how consumers respond to the interfaces provided by web-based shopping environments. As the success of electronic commerce systems lies in the wide adoption of such technologies, the success of any online buying environment may be influenced by the first impression consumers have of the interface provided (e.g., Hoque and Lohse, 1999). Moreover, the design of web interfaces that evoke a positive emotional response is also important, as such interfaces will encourage consumers to return to a particular website, a matter of critical importance to online retailers. This study aims to examine how the configuration of a web-based shopping environment influences the emotional states of consumers and how these emotions subsequently affect their purchasing intentions.

\section{Literature review and hypotheses}

While the Mehrabian-Russell framework specifies the relationships between intervening variables and response outcomes, it leaves the issue of an appropriate stimulus taxonomy largely untouched. Drawing guidance from information theory, Mehrabian and Russell (1974) proposed a general measure of environmental stimulation that would be applicable across many physical and social settings, that was termed the information rate, or "load" 
of an environment. However, since the work of Donovan and Rossiter (1982), researchers have largely explored how separate and distinct store design or layout variables such as music (e.g., Yalch and Spangenberg, 2000), colour (e.g., Bellizzi and Hite, 1992) and crowding (e.g., Machleit et al., 2000) can affect consumers in traditional retail settings. In web-based settings, authors have used the Mehrabian-Russell framework to examine the effects of variables such as high or low task relevant cues (Eroglu et al., 2003) and design and ambience factors (Mummalaneni, 2005) on online behaviour.

After encountering a stimulus, the Mehrabian-Russell framework posits that individuals will form an emotional response to the environmental stimuli just encountered. Mehrabian and Russell (1974) originally argued that three basic emotional states are responsible for mediating an individual's behaviour in environmental settings. Using the acronym PAD, these emotional states are:

1 pleasure - displeasure

2 arousal - non-arousal

3 dominance - submissiveness.

However, later studies (e.g., Russell and Pratt, 1980) led to the framework being modified, and the dimension of dominance - submissiveness is often no longer used, due to it being shown not to be a significant factor in some contexts.

Finally, the Mehrabian-Russell framework postulates that all responses to an environment can be viewed as approach or avoidance behaviours. Approach behaviours refer to a willingness to stay in, explore and return to an environment, whereas avoidance behaviours refer to deteriorated satisfaction, feelings of anxiety or boredom, and a desire to leave an environment and not return. In the retail literature, examples of approach behaviours include the increased liking of a store, a willingness to explore and spend more time in a store, the desire to return to a store at another time and an increased likelihood of spending more money than intended. While the emotional states of pleasure and arousal are posited to lead to approach or avoidance behaviours, a conditional interaction exists between these two emotional states. Specifically, in a neutral (i.e., neither pleasing nor displeasing) environment, moderate arousal enhances approach behaviours, whereas very low or very high arousal leads to avoidance behaviours. In a pleasant environment, the higher the arousal, the greater the approach behaviour, whereas in an unpleasant environment, the higher the arousal, the greater the avoidance behaviour.

Given the differences that exist between traditional and online retail environments, this paper argues that two web-specific design attributes may help predict consumer evaluations of an online retailer. These two attributes are:

1 the level of interactivity provided by an online shopping environment

2 the amount of information provided by an online shopping environment.

Of the many characteristics of the web, its most documented feature is the interactive nature of the medium. Although the concept of interactivity has been written about by a number of authors (e.g., Rafaeli, 1988; Steuer, 1995), it is a term that has been widely defined. Recent definitions of interactivity that are specific to the web have emphasised concepts such as the direction of communication, user control, and time (McMillan and Hwang, 2002), active control, two-way communication, and synchronicity (Liu and 
Shrum, 2002), and timeliness, bidirectionality, mutual controllability, and responsiveness (Yadav and Varadarajan, 2005). Following Hoffman and Novak (1996), two main types of interactivity are considered to be applicable to the web. The first, person-interactivity, is the ability for a person using the web to communicate with other individuals. The second, machine-interactivity, refers to the ability for an individual to access hypermedia content. For the purpose of this study, the term is used to refer to both types of interactivity.

Adopting this definition, increased levels of interactivity may allow consumers using an online shopping environment to gain greater control of their shopping experience (e.g., by communicating with other people using a website or by interactively accessing information from online databases). Past research (e.g., Marmorstein et al., 1992) suggests that greater control of the shopping experience is associated with increased pleasure. Further, Fiore et al. (2005) found that image interactivity was linked to increased pleasure. Therefore, increased levels of interactivity in a web-based shopping environment are also expected to have a positive relationship with pleasure. Thus:

\section{$H_{1} \quad$ There will be a positive relationship between the level of interactivity provided by a web-based shopping environment and pleasure.}

Other authors (e.g., Nasar, 1987) have also found evidence of a relationship between complexity and arousal. With higher levels of interactivity in web-based shopping environments increasing the complexity of the interface provided, it is expected that level of interactivity will be positively related to consumers' emotional state of arousal. Such a relationship was supported by Fiore et al. (2005) who found a positive relationship between image interactivity and arousal. Consequently:

\section{$\mathrm{H}_{2} \quad$ There will be a positive relationship between the level of interactivity provided by a web-based shopping environment and arousal.}

Another well-documented feature of the web is the ability for information to be made easily available to consumers. Extending this point-of-view, Cook and Coupey (1998) argued that the increased availability of information on the web has the potential to result in more knowledgeable consumers, who are then able to make better quality decisions, who will then experience greater satisfaction with any purchases they make. To help understand how the amount of information provided by an online shopping environment may affect consumer behaviour, this study draws from the literature that has examined the effects of information load on consumer decision-making in traditional (e.g., Jacoby et al., 1974; Malhotra, 1982) and online (e.g., Lee and Lee, 2004) settings. Building on the assertion that information load should be based upon the quantity of information provided on a per brand basis (e.g., Wilkie, 1974), amount of information is defined in this study as the number of attributes provided on a per-product basis by an online shopping environment.

More information can be made available at the point of purchase in online rather than offline environments (Cook and Coupey, 1998). Increased information has also been found to help consumers make more informed decisions, thereby increasing their satisfaction with the purchase process (e.g., Glazer, 1991). As a result, increased amounts of information in online shopping interfaces are expected to have a positive relationship with pleasure. Therefore: 
$\mathrm{H}_{3} \quad$ There will be a positive relationship between the amount of information provided by a web-based shopping environment and pleasure.

The amount of information provided is also predicted to affect arousal when consumers interact with an online shopping environment. As noted, past research has established support for a relationship between information rate and arousal, as well as complexity and arousal. With greater amounts of information in online shopping environments increasing the aesthetic complexity of the interface provided, a positive relationship is predicted to exist between the amount of information provided and arousal. Consequently:

$\mathrm{H}_{4} \quad$ There will be a positive relationship between the amount of information provided by a web-based shopping environment and arousal.

Pleasure has been found to increase approach behaviours within traditional (e.g., Baker et al., 1992; Donovan and Rossiter, 1982; Donovan et al., 1994; Sherman et al., 1997) and online (e.g., Eroglu et al., 2003; Fiore et al., 2005; Mummalaneni, 2005) retail settings. Similarly, this study predicts that pleasure will have a positive relationship with the likelihood that a consumer would purchase items using an online shopping interface. Therefore:

\section{$\mathrm{H}_{5} \quad$ Pleasure experienced while using a web-based shopping environment will be positively related to the likelihood consumers' would purchase items from an online retailer.}

Arousal is also expected to have an effect on purchase consideration. However, based on the work of Mehrabian and Russell (1974), and later studies which have applied the Mehrabian-Russell framework to traditional retail settings (e.g., Donovan and Rossiter, 1982; Donovan et al., 1994), a conditional interaction is predicted to exist between pleasure and arousal. In pleasant online shopping environments, the higher the arousal, the more positive purchase consideration will be. Conversely, in an unpleasant web-based shopping environment, the higher the level of arousal, the less likely a consumer is to purchase items via an online shopping interface. Thus:
$H_{6} \quad$ Arousal will be positively related to the likelihood consumers' would purchase items from an online retailer in pleasant web-based shopping environments, but inversely related in unpleasant web-based shopping environments.

\section{Methodology}

For the purpose of this study, a web-based experiment employing a $3 \times 3$ between-subjects factorial design was used, where three levels of interactivity (i.e., low, medium and high), and three levels of amount of information (i.e., low, medium and high) were manipulated as independent variables, thus producing nine experimental conditions. The stimuli for this experiment consisted of a fictitious online store for digital cameras, a product category of moderate involvement to web users. The goal of this study 
was to manipulate the level of interactivity and the amount of information a respondent encountered while browsing the store, while holding all other factors constant. In the experiment, respondents were able to view four pages of the store website, including:

1 an initial welcome or front-page to the website

2 a product catalogue page listing the camera models sold

3 two product information pages for two featured models of digital cameras.

Before filling out the questionnaire at the end, a fifth (filler task) page was provided, where respondents were made to read a short guide about consumer rights when purchasing goods via the internet.

The website used in this study was configured to display three levels of interactivity, ranging from a static website with few opportunities for interactivity in the low condition, to a website with extensive opportunities for both person- and machine-interactivity in the high condition (e.g., respondents were able to subscribe to a mailing list, use a product search engine, participate in an online discussion forum with other users, etc.). The amount of information manipulations occurred in the third and fourth (product information) pages of the experimental website. In the low condition, only a one line product description was provided, while ten and 37 product attributes (i.e., technical specifications) were provided in the medium and high conditions, respectively.

For this study, a self-recruited convenience sample of web users aged 18 years or over was sought. Participants were invited to take part in this study through announcements made to USENET newsgroups, mailing lists and web-based discussion boards. Upon accessing the experimental website, participants were first shown an introduction page. This page thanked participants for accessing the online study, provided the expected time it would take to complete the study and outlined the browser (software) requirements needed to take part. Next, participants were provided with a web page outlining the instructions for the study. This instruction page informed participants that they would be asked to visit and evaluate a proposed shopping website for digital cameras that was still under development. Participants were also told how to navigate through the site, and that although the site they were about to visit was not yet fully operational, that they should not let this influence their opinion of the website in general. After clicking on a link at the bottom of the instruction page, participants were then randomly assigned to one of the nine experimental conditions.

The experimental website was left open for a three-week period, and at the end of this time, 396 questionnaires were submitted. Of these, 379 questionnaires were suitable for inclusion into the final sample. To satisfy the assumptions of statistical techniques such as the Analysis of Variance (ANOVA), and to avoid any problems caused by unequal cell sizes (Tabachnick and Fidell, 1996), 19 cases were randomly deleted, thereby creating a total sample size of 360 (with 40 subjects per experimental condition). In terms of sociodemographic characteristics, $62 \%$ of respondents were male, $64 \%$ were between 18-34 years old, $69 \%$ held some level of university qualification, with $63 \%$ also indicating that their annual household income was at the upper-third of the national average. In terms of internet expertise, $72 \%$ of respondents used the internet for more than ten hours a week, 93\% had been using the internet for two years or more and $67 \%$ perceived themselves as being experts at using the internet. 
Scale items for the measures used in this study are provided in Table 1. The manipulation checks for perceived level of interactivity and amount of information were measured using Likert-type scales $(1=$ strongly disagree, $7=$ strongly agree). All other constructs examined in this study were measured using scales adapted from the extant literature. The emotional states of pleasure and arousal were measured using the nine-point semantic differential scales developed by Mehrabian and Russell (1974), with six items being used to measure each emotional state. The likelihood that a respondent would purchase a product from the website was measured using a four-item seven-point semantic differential scale adapted from MacKenzie et al. (1986) that had also previously been used in a web-based setting (e.g., Bruner and Kumar, 2000).

Table 1 Means, standard deviations and reliabilities of measures used

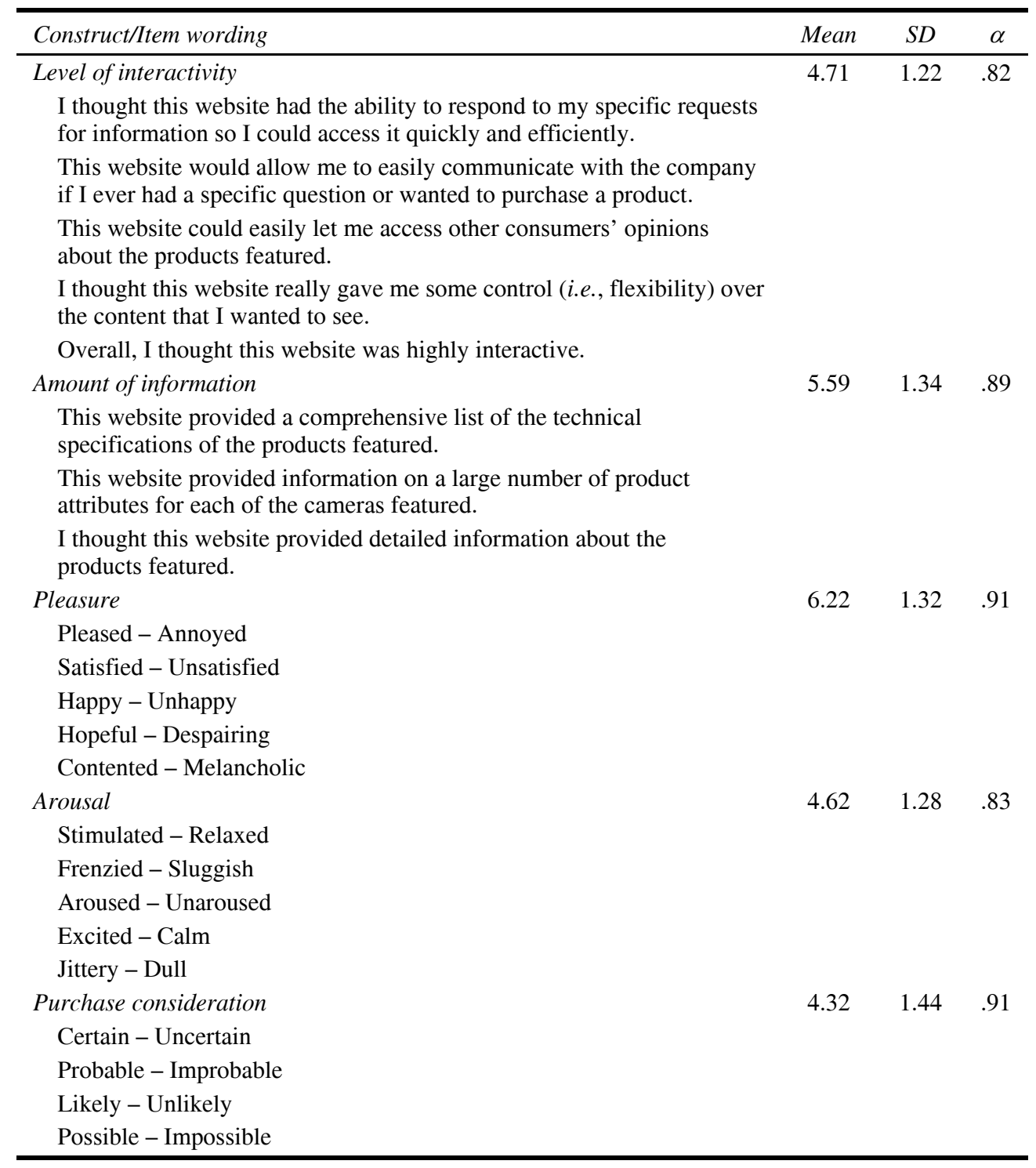




\section{Results}

Principal components analysis (with Varimax rotation) was used to assess the underlying structure of the measures used. Measures were then assessed for their internal consistency using the Cronbach alpha reliability procedure (Cronbach, 1951). Table 1 reports the means, standard deviations and reliabilities of all the measures used in this study. Perceived measures of the two independent variables were found to be both unidimensional and reliable. Analysis of the 12 pleasure and arousal items produced two factors, although due to mixed loadings, two items were subsequently dropped. The two factors supported the theoretical structure outlined by Mehrabian and Russell (1974) and were reliable. The purchase consideration measure was unidimensional and reliable.

Results of the ANOVA analyses indicated that both experimental manipulations were successful. Post-hoc Scheffe tests also confirmed that significant differences occurred between the mean scale ratings given to all three levels of the level of interactivity $(F(2,357)=47.82, p<0.01, M($ low $)=3.99, M($ medium $)=4.79, M($ high $)=5.36)$ and amount of information $(F(2,357)=78.44, p<0.01, M($ low $)=4.61, M$ (medium $)=5.80$, $M($ high $)=6.38)$ manipulations. To examine the effects of level of interactivity and amount of information on pleasure and arousal, a $3 \times 3$ between-subjects ANOVA was performed. The factors used were the three levels of level of interactivity and amount of information. Results of this analysis are presented in Table 2.

Table 2 Results of ANOVA analysis for pleasure and arousal

\begin{tabular}{lrrrrr}
\hline Source and dependent variable & \multicolumn{1}{c}{$S S$} & $d f$ & $M S$ & $F$ & $p$ \\
\hline Level of interactivity & & & & & \\
$\quad$ Pleasure & 18.32 & 2 & 9.16 & 5.51 & 0.00 \\
$\quad$ Arousal & 4.50 & 2 & 2.25 & 1.39 & 0.25 \\
Amount of information & & & & & \\
$\quad$ Pleasure & 6.53 & 2 & 3.27 & 1.97 & 0.14 \\
$\quad$ Arousal & 3.30 & 2 & 1.65 & 1.02 & 0.36 \\
Int $^{a} \times$ Amo \\
Pleasure & & & & & \\
$\quad$ Arousal & 13.69 & 4 & 3.42 & 2.06 & 0.09 \\
Error term & 9.01 & 4 & 2.25 & 1.39 & 0.24 \\
$\quad$ Pleasure & & & & & \\
$\quad$ Arousal & 582.99 & 351 & 1.66 & & \\
Total & 567.05 & 351 & 1.62 & & \\
$\quad$ Pleasure & & & & & \\
$\quad$ Arousal & 14573.72 & 360 & & & \\
\hline
\end{tabular}

Notes: a: Level of interactivity (Int); b: Amount of information (Amo).

Level of interactivity had a significant effect on pleasure, but did not have an effect upon arousal. Amount of information did not have an effect on either of the two emotional states, and no interaction effects between the two independent variables were found for pleasure or arousal. $H_{1}$ and $H_{3}$ predicted that a positive relationship would exist 
between both independent variables and pleasure. Using trend analysis, the effect of level of interactivity on pleasure was linear $(F($ linear $)=10.64, p<0.01, M($ low $)=5.67$, $M($ medium $)=6.18, M($ high $)=6.72)$, thus providing support for $H_{l}$, but not for $H_{3}$. The amount of information provided did not have an effect on pleasure or arousal, and $H_{2}$ and $\mathrm{H}_{4}$ were not supported.

To address $H_{5}$ and $H_{6}$, the approach employed by Donovan and Rossiter (1982) and Donovan et al. (1994) was followed. Two regression analyses: one for respondents who thought the experimental website provided a pleasant online shopping environment (i.e., pleasure scores above neutral) and one for respondents who considered the online store provided an unpleasant environment (i.e., pleasure scores of neutral or less) were conducted (see Table 3).

Table 3 Results of the regression analyses (standardised betas) for the effects of pleasure and

Please provide heading for Column 1. arousal on purchase consideration

\begin{tabular}{lccc}
\hline & Pleasant $(n=291)$ & Unpleasant $(n=69)$ & Total sample $(n=360)$ \\
\hline Pleasure & $0.27^{* *}$ & $0.26^{*}$ & $0.44 * *$ \\
Arousal & $0.13^{*}$ & 0.15 & $0.14 * *$ \\
$\mathrm{R}^{2}$ & 0.11 & 0.09 & 0.27 \\
$F$ & $18.28 * *$ & $3.40 *$ & $65.73 * *$ \\
$d f$ & $(2,288)$ & $(2,66)$ & $(2,357)$ \\
\hline
\end{tabular}

Notes: $\quad * p<.05 ; * * p<.01$.

The split-sample regression analysis provided only moderate support for the conditional pleasure-arousal interaction hypothesis. In pleasant web-based shopping environments, pleasure and arousal were found to be significant positive predictors of purchase consideration. Similarly, in unpleasant web-based shopping environments, pleasure was found to be a significant positive predictor of purchase consideration. However, results for the effect of arousal were not consistent with the expectations of the conditional pleasure-arousal interaction hypothesis. Instead, arousal was again a positive predictor of purchase consideration, although this result was not significant. Finally, results for the total sample (i.e., both pleasant and unpleasant environments) showed that pleasure and arousal were significant positive predictors of purchase consideration. Thus, strong support was found for a positive relationship between pleasure and purchase consideration $\left(H_{5}\right)$. However, arousal was found to be only a positive predictor of purchase consideration, providing only partial support for $H_{6}$, which predicted a conditional pleasure-arousal interaction.

\section{Discussion and conclusions}

The finding of a linear relationship between level of interactivity and pleasure suggests that, whenever possible, online retailers should maximise the interactive features provided on their website. Although such an assertion needs to be further examined in future research, the findings of this study also add support to Marmorstein et al. (1992) that greater control of the shopping experience is associated with increased consumer pleasure. 
The results for arousal outlined in this study raise questions about the applicability of this construct for use in the study of web-based shopping environments. Specifically, and contrary to the hypotheses outlined, reported ratings for arousal were found to be similar at all levels of each experimental condition. While further research is required to examine the validity of the arousal construct for use in computer-mediated settings, it is possible that this emotional state may have limited utility in helping understand consumer behaviour in web-based shopping environments. However, a caveat exists in that the majority of respondents who participated in this study were experienced users of the web. It is possible that the ability for online shopping environments to evoke arousal in consumers may be dependent on the previous level of experience an individual has with web-based shopping environments. While such a relationship was not examined in this study, this assertion is consistent with suggestions made by Mehrabian and Russell (1974) about the interrelationship between novelty and arousal, and also provides possible direction for future research efforts.

The effects of pleasure and arousal on purchase consideration provided the final area of interest in this study. A conditional pleasure-arousal interaction was hypothesised for purchase consideration; however, only mixed support was found for this hypothesis. Specifically, in both pleasant and unpleasant online shopping environments, the direction of the relationship between arousal and purchase consideration was positive, rather than the negative direction hypothesised in unpleasant environments. However, this mixed support for the conditional pleasure-arousal interaction hypothesis is consistent with research findings in bricks-and-mortar retail settings (e.g., Donovan and Rossiter, 1982; Donovan et al., 1994), and raises questions about the applicability of this conditional interaction in retail environments. Strong support was found for pleasure acting as a positive predictor of purchase consideration, thereby suggesting that it is essential for online retailers to determine what aspects of a web-based shopping environment enhance the pleasure experienced by consumers while navigating their website.

Some limitations were evident with this research. First, a fictitious retail website was used in this study, and many of the features that respondents would usually expect to find in a real-world website were not fully replicated. The second limitation relates to the issue of selection bias. As stated, participants were recruited using USENET newsgroups, mailing lists and web-based discussion boards, meaning only online respondents were able to participate in this study, and that self-selection bias may limit the generalisability of the findings presented. Thirdly, it is possible that different results may have been found for different types of retailers (e.g., supermarkets, bookstores, etc.). Thus, care should be taken in generalising the results of this study to all types of online retailers, and replication of this study to different retailer types is required. The final limitation relates to the one-shot nature of the experimental design used in this study. Specifically, respondents typically took part in this study over a relatively short timeframe (between 10-15 min). Thus, repeat visits to the experimental website may have produced a maturation effect, where respondents would be able to provide a more accurate evaluation of the website. For example, in the high interactivity condition, respondents may have required multiple exposures before becoming fully accustomed to the website and all of the navigational opportunities available.

Although the two emotional states of pleasure and arousal were used in this study, the application of other emotional typologies to research within web-based shopping environments provides a direction for future research. For example, while authors (e.g., Havlena and Holbrook, 1986) have supported the use of the Mehrabian-Russell 
dimensions of pleasure and arousal, other emotional typologies (e.g., Izard, 1977; Plutchik, 1980) have proven to be suitable for research in consumer behaviour settings. The issue of what other aspects of a web-based shopping environment are able to evoke emotional responses provides another direction for future research. In this study, both level of interactivity and amount of information were posited to affect the emotional states of pleasure and arousal. However, when navigating a web-based shopping environment (or even websites in general), there are several features that could be included on a web page that may evoke some degree of emotional response. Future research should attempt to uncover what other features found in a website elicit emotion in consumers and what effect these affective responses have on the behaviour of consumers when interacting with an online shopping interface.

\section{Acknowledgements}

Funding for this project was provided in part by the University of Canterbury and Web-Lab.

\section{References}

Baker, J., Levy, M. and Grewal, D. (1992) 'An experimental approach to making retail store environmental decisions', Journal of Retailing, Vol. 68, No. 4, pp.445-460.

Bellizzi, J.A. and Hite, R.E. (1992) 'Environmental color, consumer feelings, and purchase likelihood', Psychology \& Marketing, Vol. 9, No. 5, pp.347-363.

Bruner, G.C. and Kumar, A. (2000) 'Web commercials and advertising hierarchy-of-effects', Journal of Advertising Research, Vol. 40, Nos. 1-2, pp.35-42.

Cook, D. and Coupey, E. (1998) 'Consumer behavior and unresolved regulatory issues in electronic marketing', Journal of Business Research, Vol. 41, No. 3, pp.231-238.

Cronbach, L.J. (1951) 'Coefficient alpha and the internal structure of tests', Psychometrika, Vol. 16, No. 3, pp.297-334.

Donovan, R.J. and Rossiter, J.R. (1982) 'Store atmosphere: an environmental psychological approach', Journal of Retailing, Vol. 58, No. 1, pp.34-57.

Donovan, R.J., Rossiter, J.R., Marcoolyn, G. and Nesdale, A. (1994) 'Store atmosphere and purchasing behavior', Journal of Retailing, Vol. 70, No. 3, pp.283-294.

Eroglu, S.A., Machleit, K.A. and Davis, L.M. (2003) 'Empirical testing of a model of online store atmospherics and shopper responses', Psychology \& Marketing, Vol. 20, No. 2, pp.139-150.

Fiore, A.M., Jin, H-J. and Kim, J. (2005) 'For fun and profit: hedonic value from image interactivity and responses toward an online store', Psychology \& Marketing, Vol. 22, No. 8, pp.669-694.

Glazer, R. (1991) 'Marketing in an information intensive environment: strategic implications of knowledge as an asset', Journal of Marketing, Vol. 55, No. 4, pp.1-19.

Havlena, W.J. and Holbrook, M.B. (1986) 'The varieties of consumption experience: comparing two typologies of emotion in consumer behavior', Journal of Consumer Research, Vol. 13, No. 3, pp.394-404.

Hoffman, D.L. and Novak, T.P. (1996) 'Marketing in hypermedia computer-mediated environments: conceptual foundations', Journal of Marketing, Vol. 60, No. 3, pp.50-68.

Hoque, A.Y. and Lohse, G.L. (1999) 'An information search cost perspective for designing interfaces for electronic commerce', Journal of Marketing Research, Vol. 36, No. 3, pp.387-394. 
Izard, C.E. (1977) Human Emotions, New York: Plenum Press.

Jacoby, J., Speller, D.E. and Kohn, C.A. (1974) 'Brand choice behavior as a function of information load', Journal of Marketing Research, Vol. 11, No. 2, pp.63-69.

Kotler, P. (1974) 'Atmospherics as a marketing tool', Journal of Retailing, Vol. 49, No. 4, pp.48-64.

Lee, B-K. and Lee, W-N. (2004) 'The effect of information overload on consumer choice quality in an on-line environment', Psychology \& Marketing, Vol. 21, No. 3, pp.159-183.

Liu, Y. and Shrum, L.J. (2002) 'What is interactivity and is it always such a good thing? Implications of definition, person, and situation for the influence of interactivity on advertising effectiveness', Journal of Advertising, Vol. 31, No. 4, pp.53-64.

Machleit, K.A., Eroglu, S.A. and Mantel, S.P. (2000) 'Perceived retail crowding and shopping satisfaction: what modifies this relationship?', Journal of Consumer Psychology, Vol. 9, No. 1, pp.29-42.

MacKenzie, S.B., Lutz, R.J. and Belch, G.E. (1986) 'The role of attitude toward the ad as a mediator of advertising effectiveness: a test of competing explanations', Journal of Marketing Research, Vol. 23, No. 2, pp.130-143.

Malhotra, N.K. (1982) 'Information load and consumer decision making', Journal of Consumer Research, Vol. 8, No. 4, pp.419-430.

Marmorstein, H., Grewal, D. and Fishe, R.P.H. (1992) 'The value of time spent in price-comparison shopping: survey and experimental evidence', Journal of Consumer Research, Vol. 19, No. 1, pp.52-61.

McMillan, S.J. and Hwang, J-S. (2002) 'Measures of perceived interactivity: an exploration of the role of direction of communication, user control, and time in shaping perceptions of interactivity', Journal of Advertising, Vol. 31, No. 3, pp.29-42.

Mehrabian, A. and Russell, J.A. (1974) An Approach to Environmental Psychology, Cambridge, MA: MIT Press.

Mummalaneni, V. (2005) 'An empirical investigation of web site characteristics, consumer emotional states and on-line shopping behaviors', Journal of Business Research, Vol. 58, No. 4, pp.526-532.

Nasar, J.L. (1987) 'The effect of sign complexity and coherence on the perceived quality of retail scenes', Journal of the American Planning Association, Vol. 53, No. 4, pp.499-509.

Plutchik, R. (1980) Emotion: A Psychoevolutionary Synthesis, New York: Harper and Row.

Rafaeli, S. (1988) 'Interactivity: from new media to communication', in R.P. Hawkins, J. Wiemann and S. Pingree (Eds.) Advancing Communication Science: Merging Mass and Interpersonal Processes, Newbury Park: Sage Publications, pp.110-134.

Russell, J.A. and Pratt, G. (1980) 'A description of the affective quality attributed to environments', Journal of Personality and Social Psychology, Vol. 38, No. 2, pp.311-322.

Sherman, E., Mathur, A. and Smith, R.B. (1997) 'Store environment and consumer purchase behavior: mediating role of consumer emotions', Psychology \& Marketing, Vol. 14, No. 4, pp.361-378.

Steuer, J. (1995) 'Defining virtual reality: dimensions determining telepresence', in F. Biocca and M.R. Levy (Eds.) Communication in the Age of Virtual Reality, New Jersey: Lawrence Erlbaum, pp.33-56.

Tabachnick, B.G. and Fidell, L.S. (1996) Using Multivariate Statistics, New York: HarperCollins College Publishers.

Wilkie, W.L. (1974) 'Analysis of effects of information load', Journal of Marketing Research, Vol. 11, No. 4, pp.462-466.

Yadav, M.S. and Varadarajan, R. (2005) 'Interactivity in the electronic marketplace: an exposition of the concept and implications for research', Journal of the Academy of Marketing Science, Vol. 33, No. 4, pp.585-603.

Yalch, R.F. and Spangenberg, E.R. (2000) 'The effects of music in a retail setting on real and perceived shopping times', Journal of Business Research, Vol. 49, No. 2, pp.139-147. 